\title{
KPNA2 interaction with CBX8 contributes to the development and progression of bladder cancer by mediating the PRDM1/c-FOS pathway
}

Fanchang Zeng ${ }^{1}$, Liumei Luo ${ }^{2 *}$, Daoyuan Li', Juncheng Guo ${ }^{3}$ and Min Guo ${ }^{4}$

\begin{abstract}
Background: Bladder cancer ( $\mathrm{BCa}$ ) is a common malignancy characterized by high heterogeneity, yet the current treatment modalities are limited. The aim of the present investigation was to unravel the functional role of Karyopherin alpha 2 (KPNA2), a tumor facilitator identified in multiple malignancies, in the progression of BCa.

Methods: $B C a$ tissues and adjacent normal tissues were surgically resected and analyzed from patients with $B C$ to determine the expression profile of KPNA2 and Chromobox 8 (CBX8) by RT-qPCR, Western blot analysis and immunohistochemistry. The relationship among KPNA2, CBX8 and PR domain zinc finger protein 1 (PRDM1) was explored by co-immunoprecipitation and chromatin-immunoprecipitation. The functions of KPNA2, CBX8 and PRDM1 on BCa cell proliferation, migration and invasion were evaluated. Next, a nude mouse model of BCa was established for validating the roles of KPNA2, CBX8 and PRDM1 in vivo.

Results: KPNA2 and CBX8 were highly expressed in BCa and are in association with dismal oncologic outcomes of patients with $\mathrm{BCa}$. KPNA2 promoted nuclear import of $\mathrm{CBX} 8$. CBX8 downregulated PRDM1 by recruiting BCOR in the promoter region of PRDM1. Overexpression of KPNA2 promoted the malignant behaviors of BCa cells, which was counteracted by silencing of CBX8. Overexpressing PRDM1 attenuated the progression of BCa by inhibiting c-FOS expression. The tumor-promoting effects of KPNA2 via the PRDM1/c-FOS pathway were also validated in vivo.

Conclusion: Collectively, our findings attached great importance to the interplay between KPNA2 and CBX8 in BCa in mediating the development and progression of $\mathrm{BCa}$, thus offering a promising candidate target for better $\mathrm{BCa}$ patient management.
\end{abstract}

Keywords: Karyopherin alpha 2, Chromobox 8, PR domain zinc finger protein 1, Bladder cancer, Proliferation, Migration, Invasion

\section{Background}

Bladder cancer $(\mathrm{BCa})$ ranks the 10th most frequently occurring malignancy across the world with approximately 549,000 new cases diagnosed and 200,000 deaths in 2018 [1]. Currently available treatment modalities

\footnotetext{
*Correspondence: oadrmsnslr@163.com

${ }^{2}$ Department of Science and Education, Hainan General Hospital (Hainan Affiliated Hospital of Hainan Medical University), No. 19, Xiuhua Road, Xiuying District, Haikou 570311, People's Republic of China

Full list of author information is available at the end of the article
}

against $\mathrm{BCa}$ include complete resection, chemotherapy, and radiotherapy depending on the different disease subtypes. The identification of biomarker is of great important for different cancers, including colorectal cancer [2], gastric cancer [3], breast cancer [4] and chronic myeloid leukemia [5, 6]. A successful outcome of the treatment for $\mathrm{BCa}$ largely depends on early diagnosis and the availability of personalized therapy [7]. Understanding better the molecular features underlying the pathogenesis of $\mathrm{BCa}$ could thus offer new opportunities for early diagnosis and novel individualized and targeted therapies [8]. 
Nuclear-cytoplasmic transport has been indicated to play an important role in mediating cellular functions while the defects in this transport process are implicated in multiple types of cancers [9]. Karyopherin (KPN) nuclear transport receptors have emerged as critical regulators in altering the progression and outcome of cancer [10]. As one of the KPN family members, KPN alpha 2 (KPNA2) has been highlighted to be a biomarker of cancer, which participates in the translocation of diverse proteins associated with carcinogenesis [11]. For instance, KPNA2 has been demonstrated to harbor the oncogenic property of promoting malignant phenotypes of breast cancer cells [12]. The pro-tumor effects of KPNA2 have also been validated in hepatocellular carcinoma (HCC) where KPNA2 mediates the nuclear import of the transcriptional factor pleomorphic adenoma gene 1 (PLAG1) protein [13]. Moreover, there is evidence elucidating the function of aberrantly expressed KPNA2 in BCa by regulating the nuclear transportation of octamer-binding transcription factor 4 [14].

Besides, KPNA2 has been found to interact with Chromobox 8 (CBX8) in human lung adenocarcinoma cancer cells [15]. Expression of CBX8, which is a member of the polycomb family of epigenetic transcription factors, correlates with tumorigenesis in HCC and esophageal squamous cell carcinoma [16-18]. Additionally, the high expression of CBX8 has been elaborated to foreshadow unsatisfactory overall survival and disease-free survival (DFS) of patients with muscle invasive $\mathrm{BCa}$ [19]. However, the mechanistic evidence for interplay between KPNA2 and CBX8 in BCa poorly documented. To rectify this, we investigated in the current study how KPNA2 can affect the development and progression of $\mathrm{BCa}$ through its regulatory action on the translocation of CBX8. Thus, we endeavored to explore the functional interaction of KPNA2 with CBX8 in BCa in an attempt to unravel the underlying molecular mechanism of this oncogenic pathway.

\section{Materials and methods Ethics statement}

The study was conducted with approval of Ethics Committee of Ethics Committee of Hainan General Hospital. Each participant signed written informed consents prior to the study. The animal experiments were approved by the Animal Committee of Hainan General Hospital and conducted in accordance with the relevant regulations and requirements of the Institutional Animal Care and Use Committee (IACUC).

\section{In silico analysis}

The differential analysis of the microarray dataset GSE3167 $(n=50$, including 9 normal samples and 41
BCa samples) and GSE7476 ( $\mathrm{n}=13$, including 3 normal samples and $10 \mathrm{BCa}$ samples) obtained from the Gene Expression Omnibus (GEO) database (https://www.ncbi. nlm.nih.gov/gds) was performed using the "limma" package of $\mathrm{R}$ language (http://www.bioconductor.org/packa ges/release/bioc/html/limma.html). The BCa-related data from The Cancer Genome Atlas (TCGA) database (https://portal.gdc.cancer.gov/) were analyzed by means of Gene Expression Profiling Interactive Analysis (GEPIA) (http://gepia.cancer-pku.cn/). The differentially expressed genes (DEGs) were selected with |logFoldChange $\mid>1.8$ and $p<0.01$ as the threshold. A Venn diagram was plotted to screen out the most significant DEG through intersection of DEGs from the three databases, followed by survival analysis using GEPIA. Then, a protein-protein interaction (PPI) network was established using DEGs that were significantly correlated with patient survival condition with the help of String (https:// string-db.org/). The core degree of DEGs in PPI network was analyzed by Cytoscape (https://cytoscape.org/). The downstream regulatory pathway with highest core degree was predicted and verified by GEPIA database and hTFtarget (http://bioinfo.life.hust.edu.cn/hTFtarget\#!/).

\section{Clinical sample collection}

In total, 45 patients diagnosed with $\mathrm{BCa}$ in Hainan General Hospital from January 2013 to June 2015 were enrolled. The $\mathrm{BCa}$ tissues and adjacent non-cancerous tissues ( $\geq 3 \mathrm{~cm}$ from the edge of $\mathrm{BCa}$ tissues) were surgically resected, rapidly frozen in liquid nitrogen and stored at $-80{ }^{\circ} \mathrm{C}$ before use. BCa tissues were identified by histology and pathology and further classified according to criteria of The 2004 World Health Organization grading system. The clinical staging classified based on the International Union Against Cancer (UICC) TNM staging system [20]. Clinical tumor recurrence was recorded when tumor recurrence or metastasis was suggested by clinical and imaging examination. Overall Survival (OS) designated the time period between the date of randomization to the date of death due to any reason. Disease-free survival (DFS) designated the time period between the date of operation to the date of clinical tumor recurrence or death. All clinical data were analyzed using $\mathrm{R}$ language software. The data of KPNA2 and CBX8 expression were $\log _{2}$-transformed into a bivariate model. According to the median of the expression of KPNA2 and CBX8, patients with $\mathrm{BCa}$ were divided into high expression group and low expression group.

\section{Lentivirus infection}

A human bladder epithelial immortalized cell line SVHUC-1, Human BCa cell lines (T24 and BIU-87) and HEK293T cells were purchased from American Type 
Culture Collection (https://www.atcc.org/; Manassas, VA, USA) and cultured in Dulbecco's Modified Eagle Medium (DMEM, Thermo Fisher Scientific Inc., Waltham, MA, USA) containing $10 \%$ fetal bovine serum (FBS) (10100147, Gibco BRL, Invitrogen, CA, USA) at $37^{\circ} \mathrm{C}$ with $5 \% \mathrm{CO}_{2}$.

Cells at the logarithmic growth phase were detached by trypsin and dispersed into single cell suspension $\left(5 \times 10^{4}\right.$ cells $/ \mathrm{mL}$ ), which were then seeded in a 6-well plate ( $2 \mathrm{~mL} /$ well), cultured at $37{ }^{\circ} \mathrm{C}$ overnight and infected with different lentivirus. The lentivirus plasmids harboring overexpressed genes (oe-KPNA2, oe-CBX8, oePRDM1 and oe-c-FOS) were constructed on LV5-GFP and lentivirus plasmids harboring short hairpin RNA (shRNA) (sh-KPNA2-1, sh-KPNA2-2, sh-CBX8-1, shCBX8-2, sh-PRDM1-1 and sh-PRDM1-2) were constructed on pSIH1-H1-copGFP. The lentivirus plasmids harboring shRNA were synthesized by Gene Pharma Co., Ltd., (Shanghai, China). The supernatant was harvested, followed by centrifugation to remove virus particles and enable determination of virus titer determination. At $48 \mathrm{~h}$ after infection, the cells were collected and the expression levels of different genes were measured by reverse transcription quantitative polymerase chain reaction (RT-qPCR).

\section{RT-qPCR}

Total RNA was extracted from cells and tissues using Trizol (15596026, Invitrogen) and reversely transcribed to cDNA using a RT kit (RR047A, Takara, Kusatsu, Shiga, Japan). qPCR was performed with SYBR Premix EX Taq kit (RR420A, Takara) in a real-time fluorescence qPCR instrument (ABI7500, ABI, Foster City, CA, USA). The relative expression level was analyzed by $2-\Delta \Delta \mathrm{Ct}$ method with glyceraldehyde-3-phosphate dehydrogenase (GAPDH) serving as the loading control. Primers for KPNA2, CBX8, PRDM1 and c-FOS were synthesized by Shanghai Sangon Biotech Co., Ltd., (Shanghai, China) as shown in Table 1.

\section{Western blot analysis}

Total protein was extracted from cells and tissues by high-effective Radio Immunoprecipitation Assay Lysis Buffer (R0010, Solarbio Life Sciences Co., Ltd., Beijing, China) in strict accordance with the manual. Nucleoprotein and cytoplasmic protein were prepared using cytoplasm and nucleus protein extraction kits (Thermo Fisher Scientific). The protein concentration was determined using a bicinchonininc acid protein assay kit (20201ES76, Yeasen Biotech Co., Ltd., Shanghai, China). The proteins were separated by $10 \%$ sodium dodecylsulphatepolyacrylamide gel electrophoresis and wet-transferred onto the polyvinylidene fluoride membrane (Millipore,
Table 1 Primer sequences for RT-qPCR

\begin{tabular}{ll}
\hline Gene & Primer sequences \\
\hline KPNA2 (human) & F: 5'-CTGGGACATCAGAACAAACCAAG-3' \\
R: 5'-ACACTGAGCCATCACCTGCAAT-3' & F: 5'-CCTTCGAAACATGGGTTTGT-3' \\
CBX8 (human) & R:-CTGGGCTTGTCATCCACTCT-3' \\
PRDM1 (human) & F: 5'-CCCTCATCGGTGAAGTCTA-3' \\
& R: 5'-ACGTAGCGCATCCAGTTG-3' \\
C-FOS (human) & F: 5'-AGAATCCGAAGGGAAGGAA-3' \\
U6 & F: 5'-CGGCTCCTTCAGCAGGTTGG-3' \\
& R: 5'-CCAGTGCAGGGTCAGCGTTCCAT-3' \\
GAPDH & F: 5'-CACCCACTCCTCCACCTTTG-3' \\
& R: 5'-CCACCACCCTGTTGCTGTAG-3'
\end{tabular}

RT-qPCR: reverse transcription quantitative polymerase chain reaction; $\mathrm{F}$ : forward; R: reverse; KPNA2: karyopherin a2; CBX8: Chromobox 8; PRDM1: PR domain zinc finger protein 1; c-FOS: Cellular oncogene fos; GAPDH: glyceraldehyde-3-phosphate dehydrogenase

Billerica, MA, USA) in a wet manner. The membrane was blocked in $5 \%$ bovine serum albumin at room temperature for $1 \mathrm{~h}$, and then probed with the following diluted primary antibodies: rabbit polyclonal antibodies to KPNA2 (ab84440, 1:1000, Abcam Inc., Cambridge, UK), CBX8 (ab70796, 1:500, Abcam), PRDM1 (ab119401, 1:1000, Abcam), c-FOS (ab190289, 1:2000, Abcam), and GAPDH (ab181602, 1:2000) at $4{ }^{\circ} \mathrm{C}$ overnight, and then re-probed with horseradish peroxidase-conjugated goat anti-rabbit immunoglobulin G (ab205718, 1:10000, Abcam) at room temperature for $1 \mathrm{~h}$. The protein bands were visualized by VILBER FUSION FX5 (Vilber Lourmat, Paris, France) and analyzed by ImageJ $1.48 \mathrm{u}$ software (National Institutes of Health, Bethesda, Maryland, USA). The relative expression was calculated as the ratio of gray value of target band to that of the internal reference (GAPDH).

\section{Immunohistochemistry (IHC)}

$\mathrm{BCa}$ tissue blocks were fixed in $4 \%$ paraformaldehyde-phosphate buffer for $12 \mathrm{~h}$ and cut into slices of $4 \mu \mathrm{m}$-thickness. The tissue slides were dewaxed with xylene, hydrated by gradient ethanol, boiled in $0.01 \mathrm{M}$ citric acid buffer solution for $15-20 \mathrm{~min}$ and cooled down to room temperature. The tissue slides were incubated with $50 \mu \mathrm{L}$ primary rabbit antibodies to KPNA2 (ab84440, 1:200, Abcam) and CBX8 (ab182627, 1:50, Abcam) at room temperature for $1 \mathrm{~h}$ and with secondary antibody goat anti-rabbit immunoglobulin G (IgG; ab6728, 1:1000, Abcam) at room temperature for another $1 \mathrm{~h}$. Then the slides were incubated with streptavidin peroxidase at $37{ }^{\circ} \mathrm{C}$ for $30 \mathrm{~min}$. Color was developed using 
3,3- $N$-diaminobenzidine (DAB) for $5 \mathrm{~min}$ and reaction was terminated by washing under running water for $10 \mathrm{~min}$. Hematoxylin was applied for 2-min counterstaining, followed by hydrochloric acid-ethanol for differentiation. The sample was dehydrated, cleared, sealed and observed under an inverted microscope.

\section{Co-immunoprecipitation (Co-IP)}

T24 cells were collected and lysed. The polyclonal antibodies to KPNA2 (ab70160, 1:100, Abcam) and CBX8 (ab182627, 1:100, Abcam) were used for Co-IP with reference to the instructions of the Pierce Classic IP Kit (Thermo Fisher Scientific). In brief, protein extracts were incubated with specific antibody or IgG (negative control) at $4{ }^{\circ} \mathrm{C}$ overnight. The immune complex was collected after incubated with protein A agarose for $2 \mathrm{~h}$ at $4{ }^{\circ} \mathrm{C}$. Following 3 washes using protein lysis buffer $(0.7 \mathrm{~mL})$, the precipitate was boiled, cooled, and analyzed by $10-12 \%$ SDS-PAGE, followed by Western blot analysis.

\section{Chromatin immunoprecipitation (ChIP)}

$\mathrm{BCa}$ cells were fixed with formaldehyde for $10 \mathrm{~min}$ to generate DNA-protein crosslink and then disrupted by Ultrasonic Cell Disruptor (UP-250, Scientz, Ningbo, Zhejiang, China), $10 \mathrm{~s}$ each at an interval of $10 \mathrm{~s}, 15$ cycles in total. The supernatant was collected through centrifugation at $4{ }^{\circ} \mathrm{C}$ for $10 \mathrm{~min}$ at $12,000 \mathrm{rpm}$ and sub-packed into 2 tubes. One tube was added with rabbit antibody to IgG (ab109489, 1: 300, Abcam) as NC and the other tube was added with antibody to CBX8 (ab182627, 1: 50, Abcam) for incubation at $4{ }^{\circ} \mathrm{C}$ overnight. The DNA-protein complex was precipitated by Protein Agarose/Sepharose. The supernatant was removed through centrifugation at $12,000 \times g$ for $5 \mathrm{~min}$. The non-specific complex was washed, followed by de-crosslinking at $65{ }^{\circ} \mathrm{C}$ overnight. DNA fragments were purified and retrieved by phenol/ chloroform. The binding between CBX8 and PRDM1 was detected by RT-qPCR using PRDM1 specific primers.

\section{Immunofluorescence}

The cells were fixed in $4 \%$ paraformaldehyde for $15 \mathrm{~min}$, penetrated by $0.5 \%$ Triton X-100 (Sangon) at room temperature for $20 \mathrm{~min}$, and blocked in $5 \%$ normal goat serum (Solarbio Science \& Technology Co., Ltd., Beijing, China) at room temperature for $30 \mathrm{~min}$. Then the cells were incubated with rabbit anti-KPNA2 (ab70160, 1:100, Abcam) and CBX8 (ab182627, 1:100, Abcam) in a humidified box at $4{ }^{\circ} \mathrm{C}$ overnight. Then the cells were incubated with Alexa Fluor 647-labeled donkey anti-rabbit IgG (ab150075, 1:400, Abcam) for $1 \mathrm{~h}$ at $37^{\circ} \mathrm{C}$ in the dark. The nucleus was stained by 4,6-diamidino-2-phenylindole (BioDee Biotechnology Co., Ltd., Beijing, China) in the dark for $5 \mathrm{~min}$. The cells were finally sealed by fluorescent mounting media and images were acquired under a fluorescence microscope (IX73, Olympus, Tokyo, Japan).

\section{Cell counting kit-8 (CCK-8) assay}

The cells were seeded in a 96-well plate at a density of $2 \times 10^{3}$ cells/well. After $24 \mathrm{~h}$-infection, cell proliferation was evaluated with a CCK-8 kit (Dojindo Laboratories, Kumamoto, Japan). The optical density value at $450 \mathrm{~nm}$ was measured by a microplate reader (Bio-Rad, Hercules, CA, USA).

\section{5-ethynyl-2'-deoxyuridine (EdU) assay}

The cells at the logarithmic growth phase were seeded in a 96-well plate at a density of $2 \times 10^{3}-4 \times 10^{4}$ cells/ well and cultured overnight. $48 \mathrm{~h}$ after infection, cells were labeled with EdU by incubated with EdU $(100 \mu \mathrm{L} /$ well) for 2-h. Cells were fixed with cell fixative $(100 \mu \mathrm{L} /$ well) for 30-min at room temperature and incubated with $2 \mathrm{mg} / \mathrm{mL}$ glycine for another $5 \mathrm{~min}$. The cells were further incubated for $10 \mathrm{~min}$ with phosphate buffer saline (PBS) containing $0.5 \%$ TritonX-100 $(100 \mu \mathrm{L} /$ well $)$, stained with $1 \times$ Apollo for $30 \mathrm{~min}$ and with $1 \times$ Hoechst 33342 for $30 \mathrm{~min}(100 \mu \mathrm{L} /$ well $)$ at room temperature in the dark. Then, fluorescent mounting media $(100 \mu \mathrm{L} /$ well $)$ was used for sealing. The number of EdU-labeled cells in 3 randomly-selected fields was counted under the fluorescence microscope. EdU labeling rate $(\%)=$ the number of positive cells/(the number of positive cells + the number of negative cells) $\times 100 \%$.

\section{Terminal deoxynucleotidyl transferase dUTP nick end labeling (TUNEL) staining}

$\mathrm{BCa}$ tissues were fixed in $4 \%$ paraformaldehyde overnight, embedded with paraffin and sliced into $5-\mathrm{mm}$ sections. Five sections were dewaxed, hydrated, and incubated with $50 \mu \mathrm{L}$ of $1 \%$ proteinase $\mathrm{K}$ dilution at $37^{\circ} \mathrm{C}$ for $30 \mathrm{~min}$. The activity of endogenous peroxidase (POD) was blocked by incubated with methanol solution supplemented with $0.3 \% \mathrm{H}_{2} \mathrm{O}_{2}$ at $37{ }^{\circ} \mathrm{C}$ for $30 \mathrm{~min}$. TUNEL reaction solution was added and incubated with sections in a humidified box at $37^{\circ} \mathrm{C}$ for $1 \mathrm{~h}$ in the dark. Then, $50 \mu \mathrm{L}$ Converter-POD was added and incubated with the sections in a wet box at $37^{\circ} \mathrm{C}$ for another $30 \mathrm{~min}$. Color was visualized by incubation with $2 \%$ $\mathrm{DAB}$ for 15 - $\mathrm{min}$ at room temperature. The reaction was terminated by adding distilled water. The sections were 
then counterstained by hematoxylin, followed by dehydration using ethanol of ascending concentrations and xylene clearing. After sealed with neutral gum, sections were observed under a microscope $(40 \times)$ in 10 randomly-selected fields. The brown nuclei were indicative of apoptotic positive cells and blue nuclei were indicative of normal cells. The apoptosis rate $=$ the number of apoptotic cells/the number of normal cells.

\section{Transwell assay}

Invasion assay was performed. The extracellular matrix (ECM) gel (E1270-1ML, Sigma-Aldrich Chemical Company, St Louis, MO, USA) was diluted to $1 \mathrm{mg} / \mathrm{mL}$ with serum-free medium. The polycarbonate membrane in the apical chambers was supplemented with $40 \mu \mathrm{L}$ ECM gel and incubated for $5 \mathrm{~h}$ at $37{ }^{\circ} \mathrm{C}$ with $5 \% \mathrm{CO}_{2}$ for polymerization, which was then incubated with DMEM (70 $\mu \mathrm{L} /$ chamber) for $0.5 \mathrm{~h}$ at $37^{\circ} \mathrm{C}$ for rehydration. BCa cells were starved for $24 \mathrm{~h}$ in serum free DMEM and re-suspended in serum free DMEM to make the final cell concentration $2.5 \times 10^{5}$ cells $/ \mathrm{mL}$. Then, $0.2 \mathrm{~mL}$ cell suspension was added into apical Transwell chambers with hydrated basement membrane while the basolateral chambers were added with $700 \mu \mathrm{L}$ pre-cold DMEM containing $10 \%$ FBS, followed by incubation under saturated humidity at $37{ }^{\circ} \mathrm{C}$ with $5 \% \mathrm{CO}_{2}$ for $24 \mathrm{~h}$. With the removal of chambers, the cells on the chambers and basement membrane were wiped out using wet cotton swab, fixed with methanol for $30 \mathrm{~min}$, stained with $0.1 \%$ crystal violet for $20 \mathrm{~min}$ and aired dried. An inverted microscope (CKX53, OLYMPUS) was used for observation at $200 \times$ within 5 randomly selected fields $(200 \times)$. The number of cells that crossed through the membrane was counted.

Migration assay was conducted. Briefly, the cells were transfected with plasmid DNA for $24 \mathrm{~h}$ and then digested into a single cell suspension. Cell concentration was adjusted to $1 \times 10^{6}$ cells $/ \mathrm{mL}$ in medium containing $1 \%$ FBS. A 24-well plate was added with 10\% FBS-containing complete medium at $600 \mu \mathrm{L} /$ well. The Transwell chamber was placed on the plate and added with the adjusted amount of cell suspension at $100 \mu \mathrm{L}$ per chamber, followed by incubation at $37^{\circ} \mathrm{C}$ for $24 \mathrm{~h}$. The chamber was removed and the medium and non-migrated cells in the chamber were wiped off using a cotton swab, then fixed with a fixative with a ratio of methanol and acetic acid of 3: 1 for 15-30 min, and air-dried. The chamber was stained with $1 \%$ crystal violet for $20 \mathrm{~min}$ and cell migration was observed under an inverted microscope (CKX53, OLYMPUS, Japan), photographed and counted. Each experiment was repeated three times.

\section{Scratch test}

The transfected cells were seeded in a 6-well plate at $5 \times 10^{5}$ cells/well. When the cell growth and confluence reached about $90 \%$, the axis of the hole was gently scratched using a sterile pipette tip. After washing with PBS to remove floating cells, the cells were added with serum-free medium and continued to culture for $0.5-1 \mathrm{~h}$ to recover the cells. The cells were photographed at $0 \mathrm{~h}$ and $24 \mathrm{~h}$, respectively, and the migration distance of the cells was measured with Image-Pro Plus Analysis software (Media Cybernetics, Silver Spring, MD, USA), and the value was averaged.

\section{Xenograft tumor in nude mice}

In total, $36 \mathrm{BALA} / \mathrm{C}$ nude mice (age: $4-5$ weeks, weight: 16-18 g) were purchased from Hunan SJA Laboratory Animal Co., Ltd., (Changsha, Hunan, China) (http:// www.hnsja.com/), and raised under specific pathogen free environment. Every 12 mice were inoculated with BCa cells $\left(5 \times 10^{3}\right.$ cells/mouse) harboring oe-NC, oe-KPNA2 or oe-KPNA2 + oe-PRDM1. The volume of xenograft tumors was measured on the 10th day after inoculation and calculated using the formula that $\mathrm{V}$ $\left(\mathrm{mm}^{3}\right)=\left(\mathrm{A} \times \mathrm{B}^{2}\right) / 2$, where $\mathrm{A}$ is the long diameter and $\mathrm{B}$ is the short diameter. The curve displaying the average volume at each time point was plotted.

\section{Statistical analysis}

Data analysis was performed using SPSS 21.0 software (IBM, Armonk, NY, USA). Measurement data were expressed as the mean \pm standard deviation unless otherwise indicated. Data in normal distribution and homogeneity of variance were compared by paired $t$-test within the group, by independent sample $t$-test between two groups and by one-way analysis of variance (ANOVA) among multiple groups, followed by Tukey's post hoc test. Data comparison at different time points was analyzed by repeated measures ANOVA, followed by Tukey's post hoc test. Kaplan-Meier was introduced to plot a survival curve, while Log-rank test was used for differential analysis. Cox proportional hazard regression model was used to evaluate the individual variables influencing the survival risk of $\mathrm{BCa}$. Wald's test was used to determine the statistical 

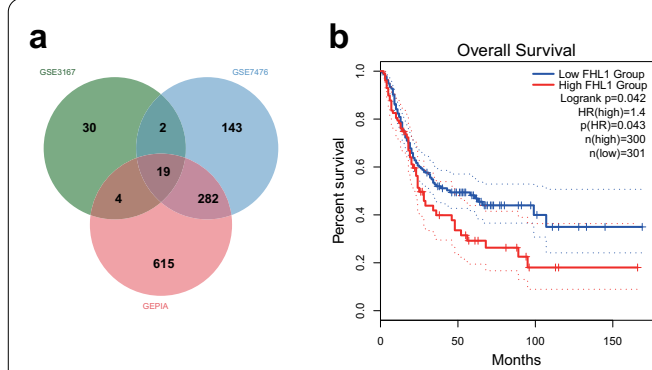

c

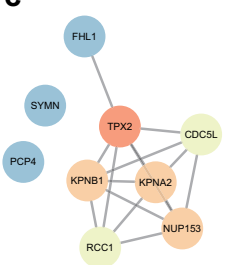

d

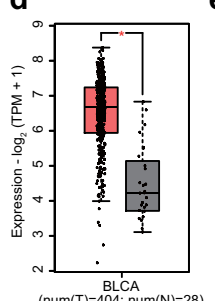

e

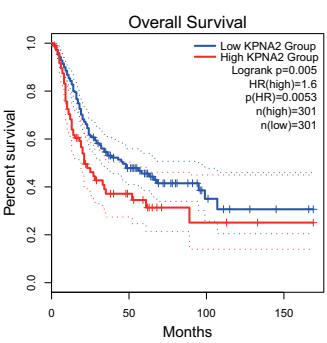

$\mathbf{f}$

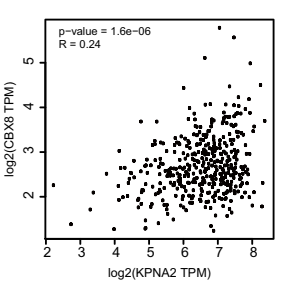
$\mathbf{f}$
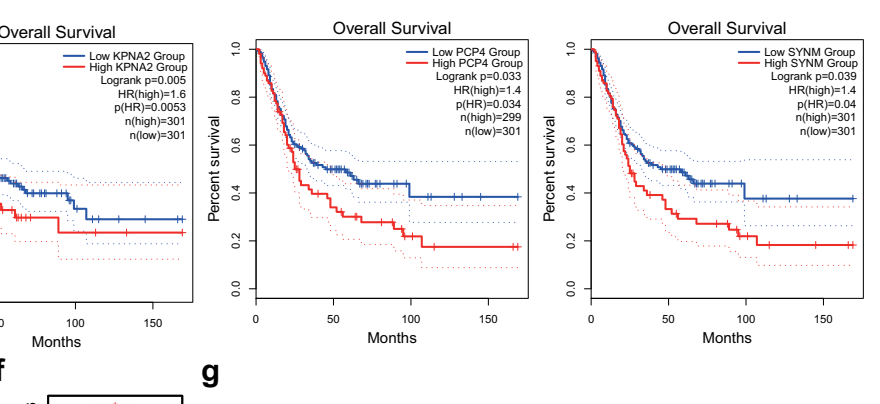

g
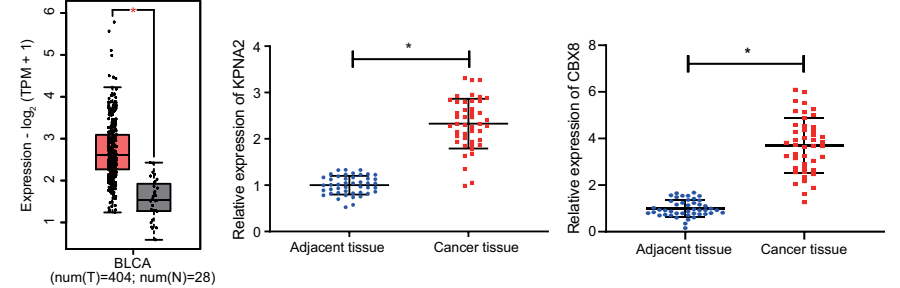

k
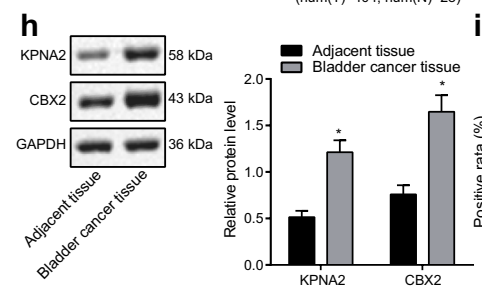

i
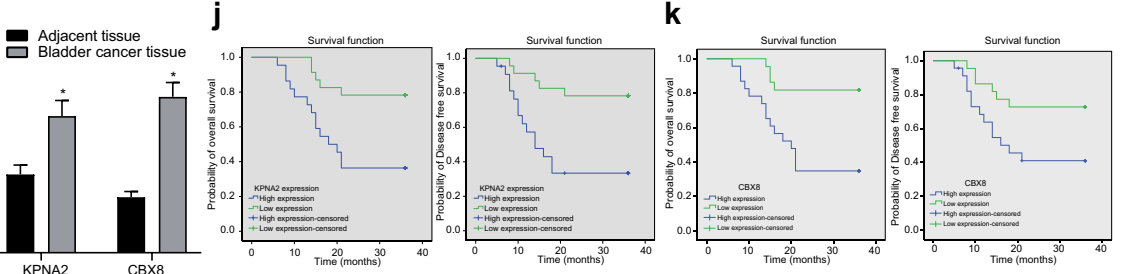

Fig. 1 High expression of KPNA2 and CBX8 in BCa is associated with dismal oncological outcomes of patients with BCa. a Venn diagram illustrating DEGs in BCa through microarray dataset GSE3167 and GSE7476 and TCGA database by GEPIA. b The survival curves displaying the correlation between the expression of KPNA2, PCP4, SYNM or FHL1 and survival of patients with BCa. c The PPI networks of KPNA2, PCP4, SYNM and FHL1. The deeper red color suggests higher core degree and the deeper blue color suggests lower core degree. $\mathbf{d}$ The box plot of KPNA2 expression in BCa samples (left red box) and normal samples (right grey box) according to GEPIA; ${ }^{*} p<0.05$ vs. normal samples. e The correlation analysis between expression of KPNA2 and CBX8 in BCa according to GEPIA $(p=1.6 \mathrm{e}-06)$. $\mathbf{f}$ The box plot of CBX8 expression in BCa samples (left red box) and normal samples (right grey box) according to GEPIA; ${ }^{*} p<0.05$ vs. normal samples. $\mathbf{g}$ The expression of KPNA2 and CBX8 in BCa tissues and adjacent non-cancerous tissues determined by RT-qPCR; ${ }^{*} p<0.05$ vs. adjacent non-cancerous tissues. $\mathbf{h}$ The expression of KPNA2 and CBX8 in BCa tissues and adjacent non-cancerous tissues determined by Western blot analysis; ${ }^{*} p<0.05 \mathrm{vs}$. adjacent non-cancerous tissues. i The expression of KPNA2 and CBX8 in BCa tissues and adjacent non-cancerous tissues identified by $\mathrm{IHC}(\times 100) ;{ }^{*} p<0.05$ vs. adjacent non-cancerous tissues. $\mathbf{j}$ The correlation between KPNA2 expression and survival condition analyzed by Kaplan-Meier. $\mathbf{k}$ The correlation between CBX8 expression and survival condition analyzed by Kaplan-Meier. $n=45$. Data comparison between BCa tissues and adjacent non-cancerous tissues was analyzed by paired $t$-test

significance of univariate and multivariate Cox regression models. A $p$ value of $<0.05$ was considered statistically significant.

\section{Results}

High expression of KPNA2 and CBX8 in BCa was correlated with poor prognosis

Through differential analysis on GEO microarray dataset GSE3167 and GSE7476 combined with GEPIA on TCGA database, 55,446 and 920 DEGs were obtained respectively and 19 DEGs were further revealed in the intersection by means of Venn diagram (Fig. 1a). Subsequently, a survival curve displaying the correlation between the expression profile of 19 DEGs and survival condition of patients with $\mathrm{BCa}$ showed 4 significantly correlated DEGs, KPNA2, PCP4, SYNM and FHL1, among which KPNA2 had the most significant correlation (Fig. 1b). Following establishment of a PPI network for the above 4 genes using String, Cytoscape was introduced for image processing, which revealed KPNA2 to be the gene with highest core degree (Fig. 1c, Table 2). Besides, KPNA2 was revealed as a significantly upregulated gene in $\mathrm{BCa}$ according to the GEPIA results (Fig. 1d, Table 3).

Further GEPIA results demonstrated a positive correlation between the expression of KPNA2 and CBX8 in BCa samples (Fig. 1e). CBX8 was confirmed to be another upregulated gene in $\mathrm{BCa}$ (Fig. 1f), which is highly suggestive of the promotive effects induced by the interplay between KPNA2 and CBX8 on the progression of $\mathrm{BCa}$. 
Table 2 Survival analysis of 19 DEGs

\begin{tabular}{llllll}
\hline Gene & $\boldsymbol{p}(\mathbf{H R})$ & HR & Gene & $\boldsymbol{p}(\mathbf{H R})$ & HR \\
\hline KPNA2 & 0.0053 & 1.6 & ACTG2 & 0.099 & 1.3 \\
PCP4 & 0.034 & 1.4 & ACTC1 & 0.1 & 1.3 \\
SYNM & 0.04 & 1.4 & MYH11 & 0.11 & 1.3 \\
FHL1 & 0.043 & 1.4 & CFD & 0.21 & 1.2 \\
SRPX & 0.051 & 1.4 & MYL9 & 0.22 & 1.2 \\
TNS1 & 0.056 & 1.4 & TOP2A & 0.35 & 0.85 \\
SPARCL1 & 0.073 & 1.3 & LMOD1 & 0.54 & 1.1 \\
TAGLN & 0.083 & 1.3 & CKS2 & 0.59 & 1.1 \\
CNN1 & 0.088 & 1.3 & MYLK & 0.62 & 1.1 \\
DES & 0.089 & 1.3 & & & \\
\hline
\end{tabular}

HR: Hazard Ratio ( $\mathrm{HR}>1$ indicates high risk gene, $\mathrm{HR}<1$ indicates low risk gene); DEG: differentially expressed genes; KPNA2: karyopherin a2; PCP4: Purkinje cell protein 4; SYNM: Synemin; FHL1: Four and a half LIM domain protein 1; SRPX: sushi repeat-containing protein X; TNS1: tensin-1; SPARCL1: Secreted protein acidic and rich in cysteine like-1; TAGLN: transgelin; CNN1: calponin-1; DES: desulfhydrase

Table 3 The core degree of significant risk genes

\begin{tabular}{ll}
\hline Gene & Degree \\
\hline FHL1 & 1 \\
PCP4 & 0 \\
SYNM & 0 \\
KPNA2 & 5 \\
\hline
\end{tabular}

FHL1: Four and a half LIM domain protein 1; PCP4: Purkinje cell protein 4; SYNM: Synemin; KPNA2: karyopherin a2

Subsequently, RT-qPCR (Fig. 1g), Western blot analysis (Fig. 1h) and IHC (Fig. 1i) were performed to determine the expression profile of KPNA2 and CBX8 in $\mathrm{BCa}$ tissues collected from patients with $\mathrm{BCa}$. We saw significantly higher expression of KPNA2 and CBX8 in $\mathrm{BCa}$ tissues than in adjacent non-cancerous tissues was observed. The correlation analysis between KPNA2 and CBX8 expression and prognosis of BCa patients through follow-up visits showed an inverse relationship, whereby patients with highly expressed KPNA2 and CBX8 exhibited poor overall survival and DFS (Fig. 1j, k). The expression of KPNA2 and CBX8 was correlated with the pathological grade, clinical stage, metastasis, and recurrence of the tumor $(p<0.05)$, but not correlated with age, gender, smoking history, and gross hematuria $(p>0.05)$ (Table 4). Taken together, these findings indicated that KPNA2 and CBX8 were highly expressed in BCa tissues and served as adverse prognostic biomarkers.
KPNA2 promoted nuclear import of CBX8

To investigate whether the nuclear translocation of CBX8 in BCa was mediated by KPNA2, KPNA2 antibody was introduced for Co-IP assay. CBX8 was detected in KPNA2 antibody-enriched precipitate of the BCa cell lines, BIU-87 and T24 (Fig. 2a). Moreover, KPNA2 antibody could enrich more CBX8 in T24 cells. Then, KPNA2 and CBX8 mRNA expression was detected in T24 and BIU-87 cell lines using RT-qPCR (Fig. 2b). The results displayed that KPNA2 and CBX8 mRNA expression was increased in T24 and BIU-87 cells relative to SV-HUC-1 cells, and their levels were most significantly upregulated in T24 cells. Therefore, T24 cells were selected for subsequent use. Next, in vitro models were developed when KPNA2 was overexpressed or knocked down in T24 cells. According to Western blot analysis, KPNA2 expression was upregulated/downregulated in response to overexpression/knockdown of KPNA2 (Fig. 2c). Following isolation of nucleoproteins and cytoplasmic proteins, Western blot analysis showed that overexpressing KPNA2 could significantly increase the expression of KPNA2 and CBX8 in the nucleus but slightly decrease the expression of CBX8 in the cytoplasm while opposite results were observed after silencing KPNA2 (Fig. 2d, e). Observation through immunofluorescence microscopy revealed accumulation of KPNA2 and CBX8 in the nucleus after overexpression of KPNA2 (Fig. 2f). To conclude, it was suggested that KPNA2 acted as positive regulator for the nuclear import of CBX8 through its binding with CBX8.

KPNA2 mediated the proliferation, migration, and invasion of $\mathrm{BCa}$ cells through regulating $\mathrm{CBX} 8$

The investigation focus was then shifted to an exploration of the functional significance of interaction between KPNA2 and CBX8 in BCa. Firstly, KPNA2 was overexpressed and CBX8 was silenced in T24 cells. RT-qPCR showed that sh-CBX8 obviously reduced the expression of CBX8 in the presence of oe-KPNA2 (Fig. 3a). Then, the proliferative capability of tumor cells was measured by CCK- 8 assays (Fig. 3b, c), migration and invasion were evaluated by Transwell assay and scratch test (Fig. 3d-g). The proliferation, migration, and invasion of $\mathrm{BCa}$ cells were significantly retarded after inhibition of KPNA2 but accelerated after overexpression of KPNA2 (Fig. 3b, d, e). Besides, silencing of CBX8 could significantly counteracted the effects of KPNA2 overexpression on the proliferation, migration, and invasion of $\mathrm{BCa}$ cells (Fig. 3c, f, g). In summary, the results elucidated that the mediation of 
Table 4 Correlation between the expression of KPNA2 and CBX8 in tissues and the characteristics of clinical cases

\begin{tabular}{|c|c|c|c|c|c|c|c|}
\hline \multirow[t]{2}{*}{ Group } & \multirow[t]{2}{*}{ Cases } & \multicolumn{2}{|c|}{ KPNA2 expression } & \multirow[t]{2}{*}{$p$} & \multicolumn{2}{|c|}{ CBX8 expression } & \multirow[t]{2}{*}{$p$} \\
\hline & & Downregulate & Upregulate & & Downregulate & Upregulate & \\
\hline \multicolumn{8}{|l|}{ Age } \\
\hline$>60$ & 15 & 5 & 10 & $>0.05$ & 7 & 8 & $>0.05$ \\
\hline$\leq 60$ & 30 & 17 & 13 & & 16 & 14 & \\
\hline \multicolumn{8}{|l|}{ Gender } \\
\hline Male & 35 & 19 & 16 & $>0.05$ & 18 & 17 & $>0.05$ \\
\hline Female & 10 & 3 & 7 & & 5 & 5 & \\
\hline \multicolumn{8}{|c|}{ TNM staging } \\
\hline G1 & 17 & 5 & 12 & $<0.05$ & 5 & 12 & $<0.05$ \\
\hline G2-G3 & 28 & 17 & 11 & & 18 & 10 & \\
\hline \multicolumn{8}{|c|}{ Clinical staging } \\
\hline $\mathrm{T} 1$ & 24 & 4 & 20 & $<0.05$ & 8 & 16 & $<0.05$ \\
\hline $\mathrm{T} 2-\mathrm{T} 3$ & 21 & 18 & 3 & & 15 & 6 & \\
\hline \multicolumn{8}{|c|}{ Smoking history } \\
\hline Yes & 28 & 15 & 13 & $>0.05$ & 13 & 15 & $>0.05$ \\
\hline No & 17 & 7 & 10 & & 10 & 7 & \\
\hline \multicolumn{8}{|c|}{ Gross hematuria } \\
\hline Yes & 30 & 15 & 15 & $>0.05$ & 16 & 14 & $>0.05$ \\
\hline No & 15 & 7 & 8 & & 7 & 8 & \\
\hline \multicolumn{8}{|c|}{ Metastasis } \\
\hline Yes & 14 & 11 & 3 & $<0.05$ & 11 & 3 & $<0.05$ \\
\hline No & 31 & 11 & 20 & & 12 & 19 & \\
\hline \multicolumn{8}{|c|}{ Recurrence } \\
\hline Yes & 19 & 15 & 4 & $<0.05$ & 14 & 5 & $<0.05$ \\
\hline No & 26 & 7 & 19 & & 9 & 17 & \\
\hline
\end{tabular}

KPNA2 in BCa cell proliferation, migration and invasion was dependent on CBX8.

\section{CBX8 downregulated PRDM1 by recruiting BCOR in the promoter region of PRDM1}

A previous report had elucidated that $\mathrm{CBX} 8$ can bind to polycomb repressive complex-BCL6 corepressor (BCOR) to suppress the transcription of positive regulatory domain 1 (PRDM1), by which lymphomagenesis is facilitated [21]. In this part of the study, we intended to explore whether PRDM1 functioned downstream of $\mathrm{CBX} 8$ in $\mathrm{BCa}$. An inverse relationship between the expression of $\mathrm{CBX} 8$ and PRDM1 in $\mathrm{BCa}$ was revealed from GEPIA (Fig. 4a) and PRDM1 was found to be poorly expressed in $\mathrm{BCa}$ (Fig. 4b). To determine the regulatory mechanism of CBX8 expression in $\mathrm{BCa}$ cells, $\mathrm{CBX} 8$ was silenced in T24 cells. Western blot analysis confirmed that $\mathrm{CBX} 8$ expression was remarkably decreased by both sh-CBX8-1 and sh-CBX8-2, and sh-CBX8-1 was selected for subsequent experiments because of its higher silencing efficiency (Fig. 4c). The enrichment of CBX8 in the
PRDM1 promoter region was then detected by ChIP assay, which revealed notably reduced enrichment of CBX8 in the promoter region of PRDM1 in response to CBX8 knockdown (Fig. 4d). For further investigation on whether CBX8 could bind to BCOR in BCa cells, a Co-IP assay was conducted in T24 cells, which showed that BCOR was enriched with CBX8 antibody compared with IgG (Fig. 4e). Then, the binding of BCOR in the PRDM1 promoter region was detected by ChIP, which showed that BCOR enrichment was dramatically reduced in the presence of sh-CBX8-1, thus suggesting that $\mathrm{CBX} 8$ was necessary for BCOR recruitment in the PRDM1 promoter (Fig. 4f). Further dual luciferase reporter assay demonstrated that silencing CBX8 could enhance the luciferase activity of PRDM1-promoter-WT (Fig. 4g). As shown in Fig. 4h, i, PRDM1 expression sharply decreased in response to $\mathrm{CBX} 8$ overexpression, and increased in response to CBX8 knockdown as measured by RT-qPCR and Western blot analysis. Collectively, CBX8 was validated to inhibit PRDM1 expression through recruitment of BCOR to the PRDM1 promoter region. 


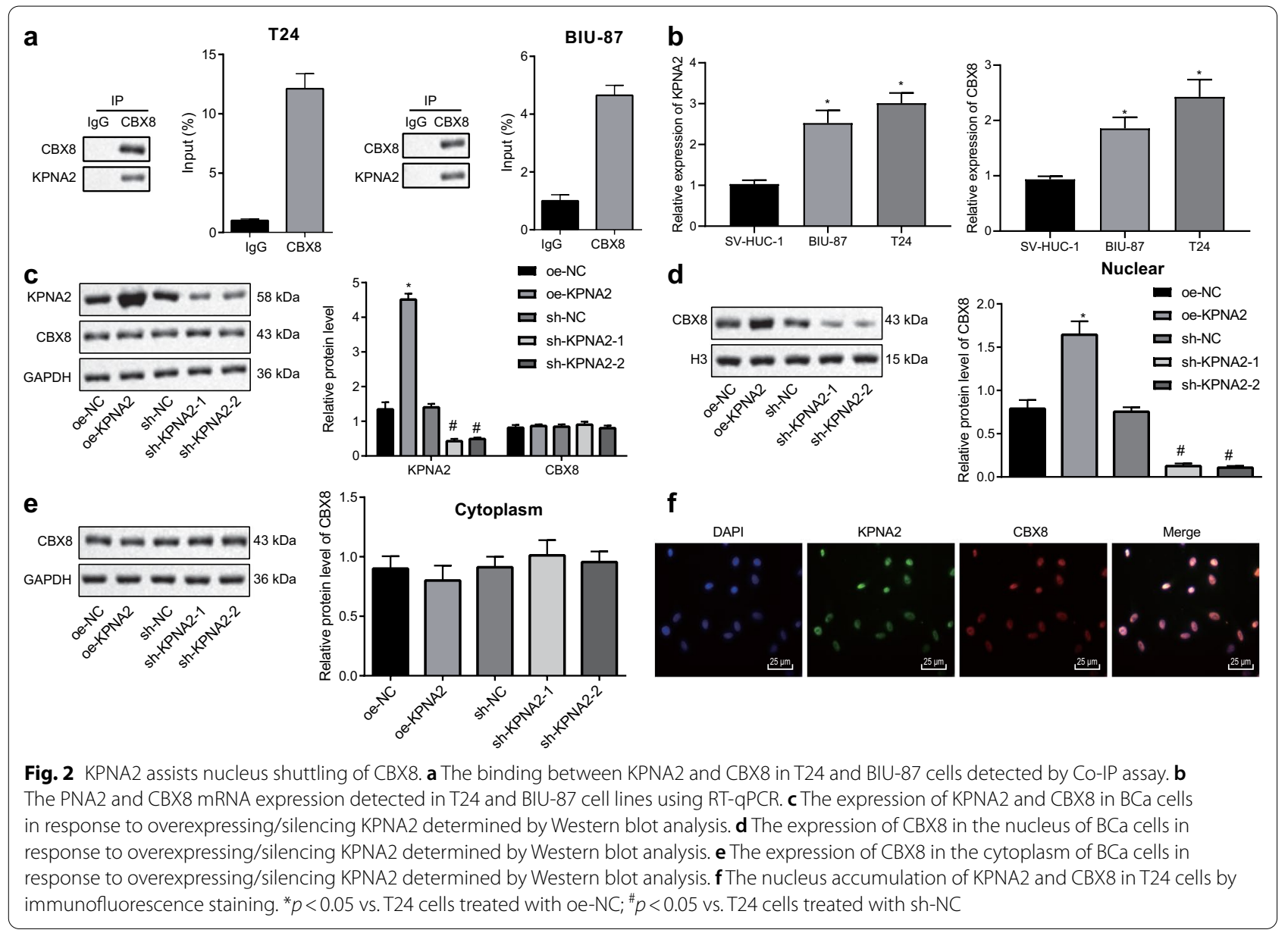

\section{PRDM1 contributed to the progression of $\mathrm{BCa}$ by inhibiting} c-FOS expression

Since PRDM1 was poorly expressed in BCa, we overexpressed PRDM1 in T24 cells. Results showed that upregulating PRDM1 suppressed cell proliferation, induced cell apoptosis and inhibited cell migration and invasion. As revealed from the hTFtarget database, PRDM1 could mediate the expression of c-FOS as a transcription factor (Fig. 5a). We overexpressed or silenced PRDM1 expression in T24 cells and then the transfection efficiency of PRDM1 were successfully validated by RT-qPCR (Fig. 5b) and Western blot analysis (Fig. 5c), among which the shPRDM1-1 with the most silencing effect was selected for subsequent use. Next, we overexpressed PRDM1 in T24 cells, and performed RT-qPCR (Fig. 5d) and Western blot analysis (Fig. 5e), which showed that c-FOS expression was significantly diminished by oe-PRDM1. Furthermore, CCK-8 assay (Fig. 5f), TUNEL staining (Fig. 5g),
Transwell assay (Fig. 5h) and scratch test (Fig. 5i) revealed that overexpressing c-FOS reversed the effects of upregulation of PRDM1 on cell proliferation, apoptosis, migration, and invasion. To conclude, PRDM1 downregulated c-FOS expression in contribution to the progression of BCa. Collectively, silencing of PRDM1 promotes the expression of C-FOS to promote the development of BCa.

\section{KPNA2/PRDM1/c-FOS axis promoted tumor growth of BCa in vivo}

Finally, we endeavored to reproduce the effects of the KPNA2/PRDM1/c-FOS axis in vivo through subcutaneous implantation of T24 cells with overexpressed KPNA2/PRDM1 into BALA/C nude mice. Results revealed that tumor volume (Fig. 6a) and weight (Fig. 6b) were significantly enhanced in the presence of oeKPNA2, whereas KPNA2 and c-FOS expression was 

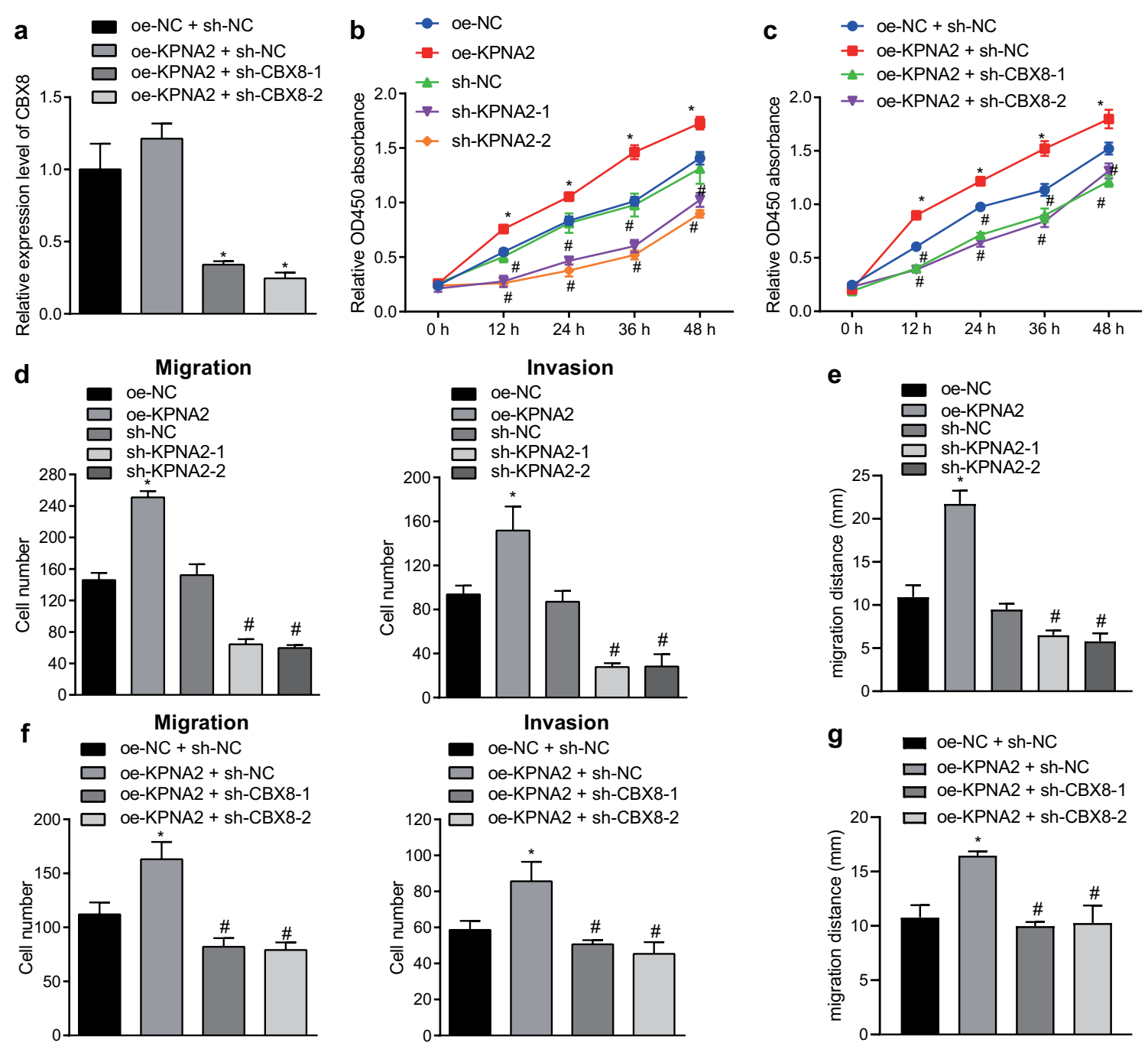

Fig. 3 CBX8 is required for the mediatory function of KPNA2 in BCa cell proliferation, migration and invasion. a The expression of CBX8 determined by RT-qPCR. ${ }^{*} p<0.05$ vs. T24 cells treated with oe-KPNA2 + sh-NC. b, $\mathbf{c}$ The proliferation of BCa cells detected by CCK-8 assay. $\mathbf{d}$ The number of migratory and invasive BCa cells detected by Transwell assay $(\times 200)$. e Migration of BCa cells detected by scratch test $(\times 200)$. f The number of migratory and invasive BCa cells detected by Transwell assay $(\times 200)$. $\mathbf{g}$ Migration of BCa cells detected by scratch test $(\times 200) .{ }^{*} p<0.05$ vs. T24 cells treated with oe-NC (panels $\mathbf{b}$ and $\mathbf{d}$ ) or oe-NC + sh-NC (panels $\mathbf{c}$ and $\mathbf{e}$ ). ${ }^{\#} p<0.05$ vs. T24 cells treated with sh-NC (panels $\mathbf{b}$ and $\mathbf{d}$ ) or oe-KPNA2 + sh-NC (panels c and e)

upregulated and PRDM1 expression was downregulated (Fig. 6c, d) accompanied by accelerated cell proliferation (Fig. 6e) and suppressed cell apoptosis (Fig. 6f). Furthermore, PRDM1 overexpression could counteract the protumor effects of KPNA2. To sum up, KPNA2 was proved to promote tumorigenesis of $\mathrm{BCa}$ in vivo through the PRDM1/c-FOS pathway.

\section{Discussion}

In recent years, research attention has been increasingly paid to the diagnostic, prognostic, and therapeutic significance of nuclear transportation in malignancies. In this context, the KPN superfamily stands out as a group of soluble transport receptors [22]. During the current investigation, we selected KPNA2 as the research target 


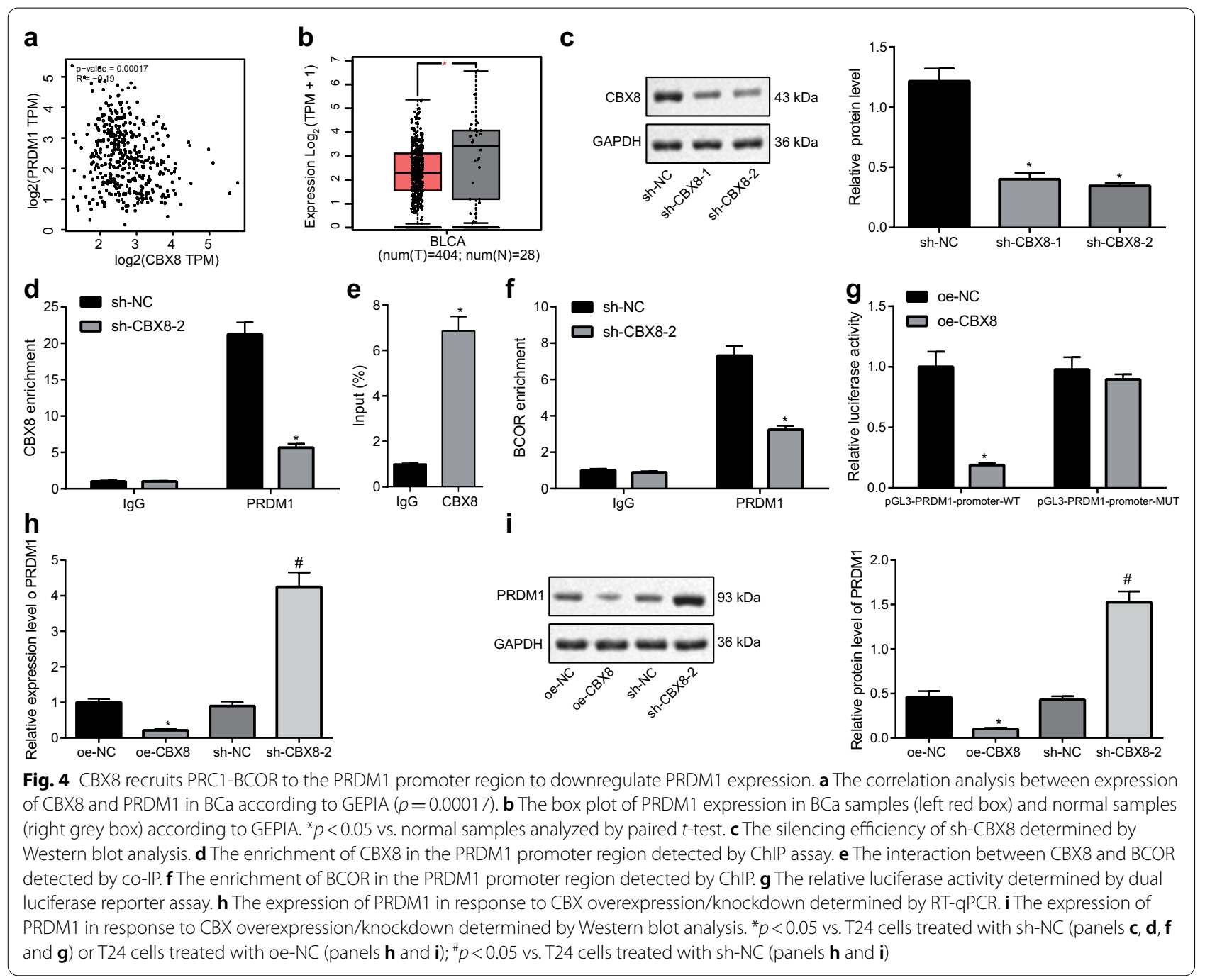

to assess its prognostic value in $\mathrm{BCa}$. Collectively, the experimental data demonstrated a detrimental contribution of KPNA2 to $\mathrm{BCa}$ in enhancing the proliferation, migration, and invasion of $\mathrm{BCa}$ cells in both in vitro and in vivo. This detrimental effect was obtained by assisting the nuclear shuttling of CBX8 and therefore regulating the PRDM1/c-FOS pathway. Results of our study suggested that high expression of CBX8 played a critical oncogenic role in the aggressiveness of urothelial carcinoma cells of the bladder by promoting cancer cell proliferation. These results suggested that CBX8 could be used as a novel biomarker predictive for muscle invasive $\mathrm{BCa}$ in patients.

An initial finding of our study was that KPNA2 and CBX8 were found to be highly expressed in BCa tissues and cell, both of which serve as independent risk factor for overall survival and DFS of patients with $\mathrm{BCa}$, indicating the clinical significance of these proteins. Largely in agreement with our present results, high expression of KPNA2 has been identified in patients with $\mathrm{BCa}$ as an independent biomarker for dismal outcomes in $\mathrm{BCa}$ patients, and to be highly suggestive of greater risk for 


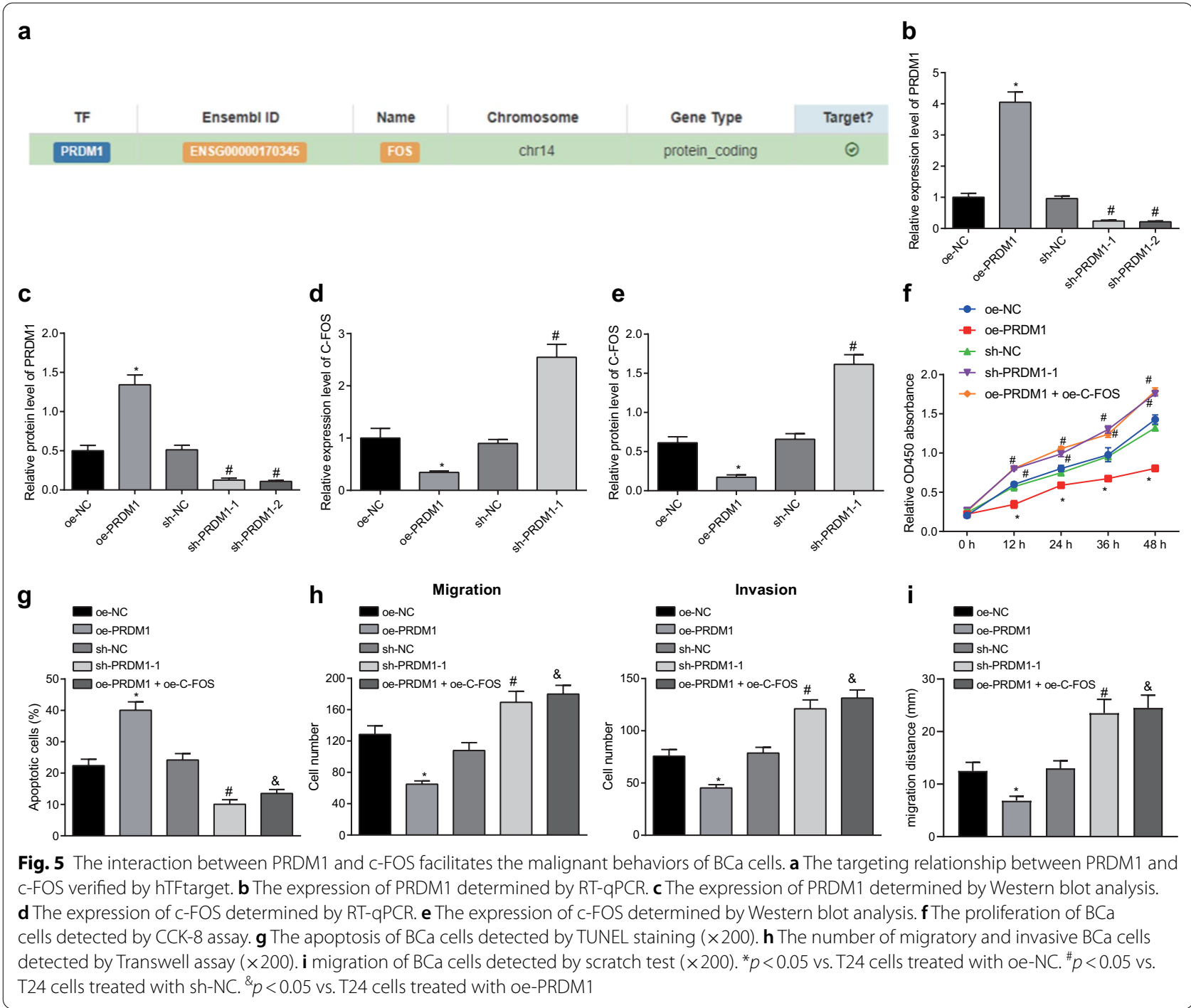

visceral metastasis and bladder recurrence after treatment [23, 24]. In the context of colorectal cancer, nonsmall-cell lung cancer and HCC, patients with high expression of KPNA2 have been reported to usually have generally unsatisfactory overall survival condition [2527]. Besides, patients with muscle invasive $\mathrm{BCa}$ exhibited high expression of CBX8 in association with reduced overall survival and DFS [19]. Accumulating evidence has revealed the involvement of CBX8 in regulatory work of different malignancies, including breast cancer, esophageal carcinoma, and HCC [28-30]. Moreover, upregulated $\mathrm{CBX} 8$ expression exerts oncogenic function on the aggressiveness of $\mathrm{BCa}$ cells by promoting the malignant prototype by repressing the p53 pathway [19] supporting the generalization of our findings. On the other hand, inhibition of CBX8 in T24 cells could counterbalance the pro-tumor effects of KPNA2, suggesting that the oncogenic role of KPNA2 in BCa was dependent on CBX8. 

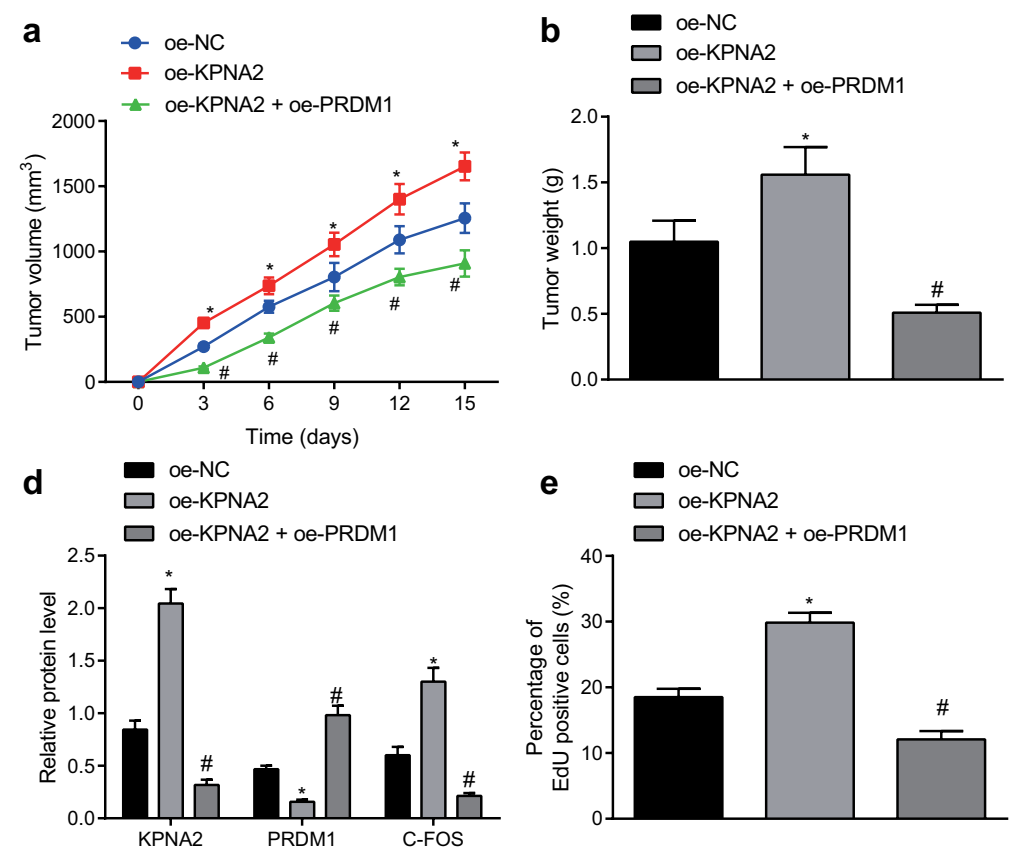
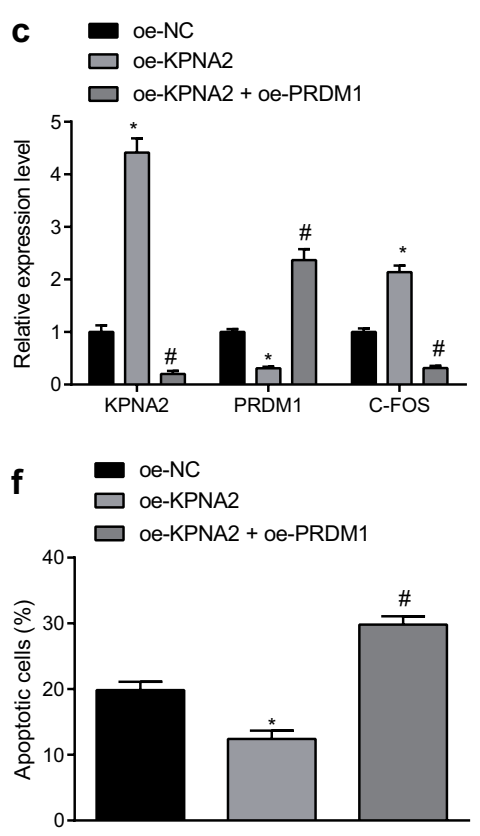

Fig. 6 KPNA2 contributes to the xenograft tumor growth of BCa in vivo by mediating the PRDM1/c-FOS pathway. a The curve displaying tumor volume at various time points. $\mathbf{b}$ The representative images of resected tumors and curve displaying tumor weight at various time points. $\mathbf{c}$ The expression of KPNA2, PRDM1 and c-FOS in transplanted tumor tissues determined by RT-qPCR. $\mathbf{d}$ The expression of KPNA2, PRDM1 and c-FOS in transplanted tumor tissues determined by Western blot analysis. $\mathbf{e}$ The tumor cell proliferation detected by EdU assay $(\times 400)$. $\mathbf{f}$ The tumor cell apoptosis detected by TUNEL assay $(\times 200) . n=12 .{ }^{*} p<0.05$ vs. nude mice bearing T24 cells treated with oe-NC. ${ }^{p} p<0.05$ vs. nude mice bearing T24 cells treated with oe-KPNA2

Furthermore, silencing of KPNA2 led to suppressed cell proliferation, migration, and invasion in vitro as well as inhibited carcinogenic potency in vivo. Concordant with our study, KPNA2 is known for its capacity to shuttle cargo proteins related to tumorigenicity between nucleus and cytoplasm [31, 32]. Concordantly, a previous study has confirmed the crosstalk between KPNA2 and $\mathrm{OCT}$ in $\mathrm{BCa}$, showing that nuclear transportation of OCT can be inhibited by silencing KPNA2 [14]. Similar to our findings, the nuclear import of PLAG1 has been illustrated to be aided by KPNA2 in hepatocellular carcinoma (HCC) and PLAG1 seemed to be partly responsible for the functional role of KPNA2 in HCC [13]. Also, the significance of KPNA2 as a nucleocytoplasmic transport protein in breast cancer has been recognized by virtue of its mediation of the subcellular localization of proteins associated with DNA damage response in breast cancer [33].

Additionally, our study showed CBX8 downregulated the PRDM1 expression by recruiting BCOR to the PRDM1 promoter region. Likewise, the interplay between CBX8 and BCOR has been documented to play a role in lymphomagenesis [21]. Downregulation of PRDM1 has been elucidated to correspond to poor prognosis and increased malignant phenotypes in lung cancer and colon cancer [34, 35]. Moreover, silencing of PRDM1 exerted promoting effects on the development of BCa by upregulating c-FOS, which is significantly upregulated in BCa [36-38]. 


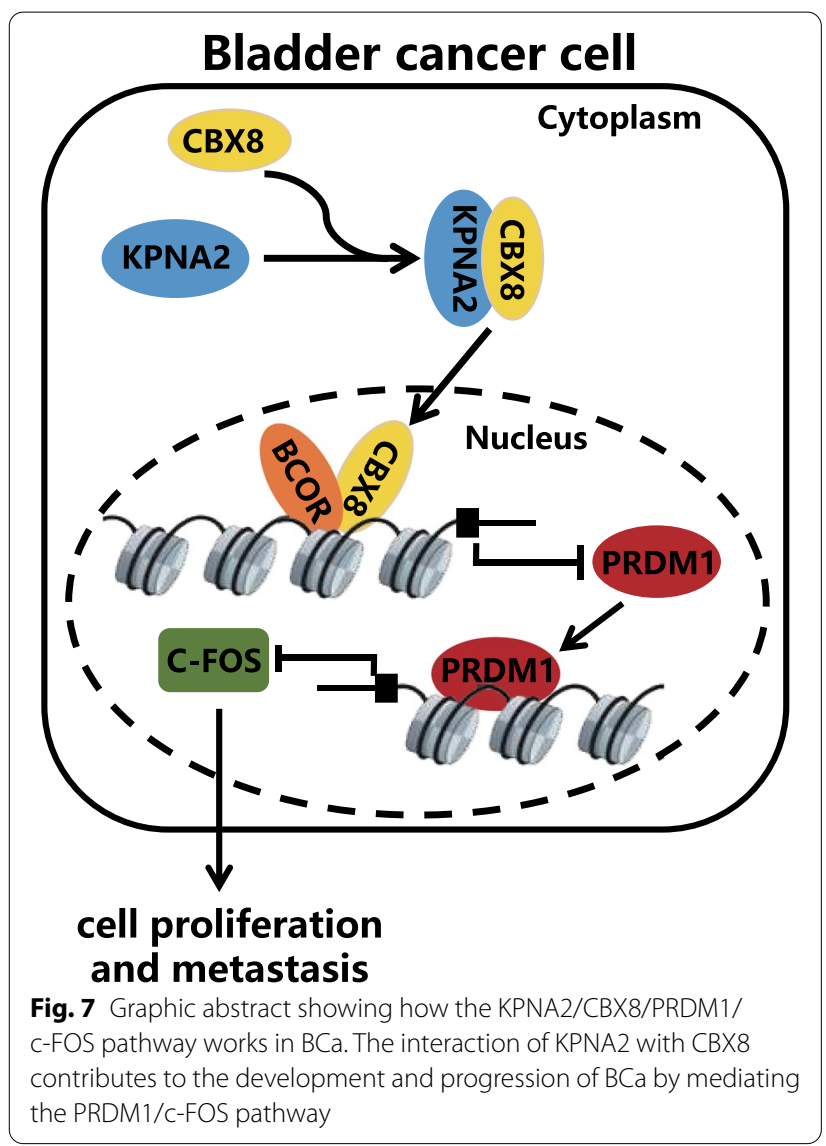

\section{Conclusion}

Collectively, our results allowed the decipherment of a functional axis whereby KPNA2 assisted the nuclear import of $\mathrm{CBX} 8$ to activate downstream effectors by recruiting $\mathrm{BCOR}$ to the promoter region of PRDM1 (see Fig. 7 for details), thus highlighting the tumor-promoting properties of KPNA2 in BCa. Our works hints at the possibility of using this axis to define a new therapeutic target to halt the development and progression of $\mathrm{BCa}$.

\section{Abbreviations}

ANOVA: Analysis of variance; BCa: Bladder cancer; CBX8: Chromobox 8; DEGs: Differentially expressed genes; DFS: Disease-free survival; ECM: Extracellular matrix; GAPDH: Glyceraldehyde-3-phosphate dehydrogenase; GEO: Gene Expression Omnibus; GEPIA: Gene Expression Profiling Interactive Analysis; KPN: karyopherin: KPNA2: KPN alpha 2: RT-qPCR: Reverse transcription quantitative polymerase chain reaction; PBS: Phosphate buffer saline; POD: Peroxidase; PPI: Protein-protein interaction; PRDM1: PR domain zinc finger protein 1.

\section{Acknowledgements}

The authors would like to acknowledge the helpful comments on this paper received from the reviewers.

\section{Authors' contributions}

FC Z and LM L designed the study. FC Z, LM L, DY L and JH G collated the data, carried out data analyses and produced the initial draft of the manuscript. $M$
G contributed to drafting the manuscript. All authors have read and approved the final submitted manuscript.

\section{Funding}

None.

\section{Availability of data and materials}

The datasets generated and analysed during the current study are available from the corresponding author on reasonable request.

\section{Ethics approval and consent to participate}

The study was conducted with approval of Ethics Committee of Ethics Committee of Hainan General Hospital. Each participant signed written informed consents prior to the study. The animal experiments were approved by the Animal Committee of Hainan General Hospital and conducted in accordance with the relevant regulations and requirements of the Institutional Animal Care and Use Committee (IACUC).

\section{Consent for publication}

Not applicable.

\section{Competing interests}

The authors declare that they have no competing interests.

\section{Author details}

${ }^{1}$ Department of Urology, Hainan General Hospital (Hainan Affiliated Hospital of Hainan Medical University), Haikou 570311, People's Republic of China.

${ }^{2}$ Department of Science and Education, Hainan General Hospital (Hainan Affiliated Hospital of Hainan Medical University), No. 19, Xiuhua Road, Xiuying District, Haikou 570311, People's Republic of China. ${ }^{3}$ Department of Urology, Central South University Xiangya School of Medicine Affiliated Haikou Hospital, Haikou 570208, People's Republic of China. ${ }^{4}$ Psychological Research Center, Hainan General Hospital (Hainan Affiliated Hospital of Hainan Medical University), Haikou 570311, People's Republic of China.

Received: 21 June 2020 Accepted: 20 January 2021

Published online: 17 March 2021

\section{References}

1. Bray F, Ferlay J, Soerjomataram I, Siegel RL, Torre LA, Jemal A. Global cancer statistics 2018: GLOBOCAN estimates of incidence and mortality worldwide for 36 cancers in 185 countries. CA Cancer J Clin. 2018;68:394-424

2. Abdel Ghafar MT, Gharib F, Abdel-Salam S, Elkhouly RA, Elshora A, Shalaby $\mathrm{KH}$, et al. Role of serum Metadherin mRNA expression in the diagnosis and prediction of survival in patients with colorectal cancer. Mol Biol Rep. 2020;47:2509-19.

3. El-Guindy DM, Wasfy RE, Abdel Ghafar MT, Ali DA, Elkady AM. Oct4 expression in gastric carcinoma: association with tumor proliferation, angiogenesis and survival. J Egypt Natl Canc Inst. 2019;31:3.

4. Abdel Ghafar MT, Gharib F, Al-Ashmawy GM, Mariah RA. Serum hightemperature-required protein A2: a potential biomarker for the diagnosis of breast cancer. Gene Rep. 2020;20:100706.

5. Habib EM, Nosiar NA, Eid MA, Taha AM, Sherief DE, Hassan AE, et al. Circulating miR-146a expression predicts early treatment response to imatinib in adult chronic myeloid leukemia. J Investig Med. 2020. https:// doi.org/10.1136/jim-2020-001563.

6. Abdelghafar M, Allam A, Darwish S. Serum HOX transcript antisense RNA expression as a diagnostic marker for chronic myeloid leukemia. Egypt $J$ Haematol. 2019;44:91

7. Kamat AM, Hahn NM, Efstathiou JA, Lerner SP, Malmstrom PU, Choi W, et al. Bladder cancer. Lancet. 2016:388:2796-810.

8. Knowles MA, Hurst CD. Molecular biology of bladder cancer: new insights into pathogenesis and clinical diversity. Nat Rev Cancer. 2015;15:25-41.

9. Kau TR, Way JC, Silver PA. Nuclear transport and cancer: from mechanism to intervention. Nat Rev Cancer. 2004:4:106-17. 
10. Cagatay T, Chook YM. Karyopherins in cancer. Curr Opin Cell Biol. 2018;52:30-42.

11. Christiansen A, Dyrskjot L. The functional role of the novel biomarker karyopherin alpha 2 (KPNA2) in cancer. Cancer Lett. 2013;331:18-23.

12. Noetzel E, Rose M, Bornemann J, Gajewski M, Knuchel R, Dahl E. Nuclear transport receptor karyopherin-alpha2 promotes malignant breast cancer phenotypes in vitro. Oncogene. 2012;31:2101-14.

13. Hu ZY, Yuan SX, Yang Y, Zhou WP, Jiang H. Pleomorphic adenoma gene 1 mediates the role of karyopherin alpha 2 and has prognostic significance in hepatocellular carcinoma. J Exp Clin Cancer Res. 2014;33:61.

14. Zhou J, Dong D, Cheng R, Wang Y, Jiang S, Zhu Y, et al. Aberrant expression of KPNA2 is associated with a poor prognosis and contributes to OCT4 nuclear transportation in bladder cancer. Oncotarget. 2016;7:72767-76.

15. Wang $\mathrm{Cl}$, Wang $\mathrm{CL}$, Wu YC, Feng HP, Liu PJ, Chang YS, et al. Quantitative proteomics reveals a novel role of karyopherin alpha 2 in cell migration through the regulation of vimentin-pErk protein complex levels in lung cancer. J Proteome Res. 2015;14:1739-51.

16. Tang B, Tian Y, Liao Y, Li Z, Yu S, Su H, et al. CBX8 exhibits oncogenic properties and serves as a prognostic factor in hepatocellular carcinoma. Cell Death Dis. 2019;10:52.

17. Xiao L, Zhou Z, Li W, Peng J, Sun Q, Zhu H, et al. Chromobox homolog 8 (CBX8) interacts with Y-Box binding protein 1 (YBX1) to promote cellular proliferation in hepatocellular carcinoma cells. Aging. 2019;11:7123-49.

18. Zhang Y, Chen H, Zhu H, Sun X. CBX8 promotes tumorigenesis and confers radioresistance in esophageal squamous cell carcinoma cells through targeting APAF1. Gene. 2019;711:143949.

19. Yuan GJ, Chen X, Lu J, Feng ZH, Chen SL, Chen RX, et al. Chromobox homolog 8 is a predictor of muscle invasive bladder cancer and promotes cell proliferation by repressing the p53 pathway. Cancer Sci. 2017:108:2166-75.

20. LiY, Zheng F, Xiao X, Xie F, Tao D, Huang C, et al. CircHIPK3 sponges miR558 to suppress heparanase expression in bladder cancer cells. EMBO Rep. 2017;18:1646-59.

21. Beguelin W, Teater M, Gearhart MD, Calvo Fernandez MT, Goldstein RL, Cardenas MG, et al. EZH2 and BCL6 cooperate to assemble CBX8-BCOR complex to repress bivalent promoters, mediate germinal center formation and lymphomagenesis. Cancer Cell. 2016;30:197-213.

22. Stelma T, Chi A, van der Watt PJ, Verrico A, Lavia P, Leaner VD. Targeting nuclear transporters in cancer: diagnostic, prognostic and therapeutic potential. IUBMB Life. 2016;68:268-80.

23. Jensen JB, Munksgaard PP, Sorensen CM, Fristrup N, BirkenkampDemtroder K, Ulhoi BP, et al. High expression of karyopherin-alpha2 defines poor prognosis in non-muscle-invasive bladder cancer and in patients with invasive bladder cancer undergoing radical cystectomy. Eur Urol. 2011;59:841-8.

24. Shi B, Su B, Fang D, Tang Y, Xiong G, Guo Z, et al. High expression of KPNA2 defines poor prognosis in patients with upper tract urothelial carcinoma treated with radical nephroureterectomy. BMC Cancer. 2015;15:380.
25. Takada T, Tsutsumi S, Takahashi R, Ohsone K, Tatsuki H, Suto T, et al. KPNA2 over-expression is a potential marker of prognosis and therapeutic sensitivity in colorectal cancer patients. J Surg Oncol. 2016;113:213-7.

26. Li XL, Jia LL, Shi MM, Li X, Li ZH, Li HF, et al. Downregulation of KPNA2 in non-small-cell lung cancer is associated with Oct4 expression. J Transl Med. 2013;11:232.

27. Guo X, Wang Z, Zhang J, Xu Q, Hou G, Yang Y, et al. Upregulated KPNA2 promotes hepatocellular carcinoma progression and indicates prognostic significance across human cancer types. Acta Biochim Biophys Sin (Shanghai). 2019;51:285-92.

28. Lee SH, Um SJ, Kim EJ. CBX8 suppresses Sirtinol-induced premature senescence in human breast cancer cells via cooperation with SIRT1. Cancer Lett. 2013;335:397-403.

29. Xiao W, Ou C, Qin J, Xing F, Sun Y, Li Z, et al. CBX8, a novel DNA repair protein, promotes tumorigenesis in human esophageal carcinoma. Int J Clin Exp Pathol. 2014;7:4817-26.

30. Zhang $\mathrm{CZ}$, Chen $\mathrm{SL}$, Wang $\mathrm{CH}$, He YF, Yang X, Xie D, et al. CBX8 exhibits oncogenic activity via AKT/beta-catenin activation in hepatocellular carcinoma. Cancer Res. 2018;78:51-63.

31. Yasuhara N, Kumar PK. Aptamers that bind specifically to human KPNA2 (importin-alpha1) and efficiently interfere with nuclear transport. J Biochem. 2016;160:259-68.

32. Han Y, Wang X. The emerging roles of KPNA2 in cancer. Life Sci. 2020:241:117140.

33. Alshareeda AT, Negm OH, Green AR, Nolan CC, Tighe P, Albarakati N, et al. KPNA2 is a nuclear export protein that contributes to aberrant localisation of key proteins and poor prognosis of breast cancer. Br J Cancer. 2015;112:1929-37.

34. Zhu Z, Wang H, Wei Y, Meng F, Liu Z, Zhang Z. Downregulation of PRDM1 promotes cellular invasion and lung cancer metastasis. Tumour Biol. 2017:39:1010428317695929.

35. Liu C, Banister CE, Weige CC, Altomare D, Richardson JH, Contreras CM, et al. PRDM1 silences stem cell-related genes and inhibits proliferation of human colon tumor organoids. Proc Natl Acad Sci USA. 2018;115:E5066-75

36. Li S, Xu X, Xu X, Hu Z, Wu J, Zhu Y, et al. MicroRNA-490-5p inhibits proliferation of bladder cancer by targeting c-Fos. Biochem Biophys Res Commun. 2013:441:976-81.

37. Lan G, Yang L, Xie X, Peng L, Wang Y. MicroRNA-490-5p is a novel tumor suppressor targeting c-FOS in human bladder cancer. Arch Med Sci. 2015;11:561-9.

38. Long Y, Wu Z, Yang X, Chen L, Han Z, Zhang Y, et al. MicroRNA-101 inhibits the proliferation and invasion of bladder cancer cells via targeting c-FOS. Mol Med Rep. 2016;14:2651-6.

\section{Publisher's Note}

Springer Nature remains neutral with regard to jurisdictional claims in published maps and institutional affiliations.

Ready to submit your research? Choose BMC and benefit from

- fast, convenient online submission

- thorough peer review by experienced researchers in your field

- rapid publication on acceptance

- support for research data, including large and complex data types

- gold Open Access which fosters wider collaboration and increased citations

- maximum visibility for your research: over 100M website views per year

At BMC, research is always in progress.

Learn more biomedcentral.com/submissions 